\title{
Development and Clinical Evaluation of a CRISPR-Based Diagnostic for Rapid Group B Streptococcus Screening
}

\author{
Lingxiao Jiang, ${ }^{1}$ Weiqi Zeng, ${ }^{1}$ Wanting Wu, ${ }^{1}$ Yingying Deng, Fusheng He, Wenli Liang, \\ Mingyao Huang, Hong Huang, Yongjun Li, Xiaorui Wang, Hang Su, Shilei Pan, Teng X ${ }^{1}$
}

\begin{abstract}
Vertical transmission of group B Streptococcus (GBS) is among the leading causes of neonatal illness and death. Colonization with GBS usually is screened weeks before delivery during pregnancy, on the basis of which preventive measures, such as antibiotic prophylaxis, were taken. However, the accuracy of such an antenatal screening strategy has been questionable because of the intermittent nature of GBS carriage. We developed a simple-touse, rapid, CRISPR-based assay for GBS detection. We conducted studies in a prospective cohort of 412 pregnant women and a retrospective validation cohort to evaluate its diagnostic performance. We demonstrated that CRISPR-GBS is highly sensitive and offered shorter turnaround times and lower instrument demands than PCRbased assays. This novel GBS test exhibited an overall improved diagnostic performance over culture and PCRbased assays and represents a novel diagnostic for potential rapid, point-of-care GBS screening.
\end{abstract}

Croup B Streptococcus (GBS) is a common com$\exists$ mensal bacteria of vaginal flora with reported carriage rates of $4 \%-40 \%(1-3)$. Vertical transmission of (GBS) through fetal aspiration of infected amniotic fluid or during birth canal passage has been considered one of the most important causes of neonatal illness and death $(3,4)$. GBS colonization during pregnancy has been a leading cause of severe neonatal

Author affiliations: Zhujiang Hospital, Southern Medical University, Guangzhou, China (L. Jiang, Y. Deng, W. Liang, M. Huang); Vision Medicals Center for Medical Research, Shenzhen, China (W. Zeng, W. Wu, H. Huang, Y. Li, X. Wang, H. Su, T. Xu); Key Laboratory of Animal Gene Editing and Animal Cloning in Yunnan Province and College of Veterinary Medicine, Yunnan Agricultural University, Kunming, China (W. Zeng, T. Xu); Zhujiang Hospital, Southern Medical University, Guangzhou (S. Pan) infectious diseases, including sepsis, pneumonia, and meningitis $(5,6)$. Early onset neonatal infections can be prevented in most cases by providing intrapartum antibiotic prophylaxis to the colonized mother (7). However, GBS carriages are often intermittent, and the rate of GBS colonization varies during pregnancy $(1,8)$. On the other hand, use of antibiotic prophylaxis solely relying on risk assessment leads to unnecessary treatment in many women. Therefore, determination of colonization at the time of delivery is crucial for the prevention of neonatal infection (9).

Culture-based methods remain the most commonly used screening practice and the standard for GBS detection; however, because of technical limitations, including turnaround time, pregnant women are usually screened for GBS at 35-37 weeks of gestation (6). As many studies have pointed out, the predictive value of GBS decreases as the interval time increases between screening and delivery $(10,11)$. These studies underlie the needs for a more rapid and sensitive diagnostic for intrapartum GBS screening.

CRISPR/Cas has been widely used as a programmable tool for gene editing and other in vivo applications since 2013 (12-14). However, recently, the collateral, promiscuous cleavage activities of a unique group of Cas enzymes were discovered and harnessed for in vitro nucleic acid detection (15-17).

To address the unmet clinical needs for GBS screening, we developed CRISPR-GBS, a novel CRIS$\mathrm{PR} /$ Cas13-based in vitro diagnostic assay, and conducted a prospective cohort study and a validation study in $>400$ clinical cases to evaluate its diagnostic performance among different technology platforms, including culture and PCR-based methods. Our findings demonstrate that CRISPR-GBS is rapid and easy-

${ }^{1}$ These authors contributed equally to this article. 
to-use, having a low instrument requirement and a level of sensitivity that surpasses PCR-based assays.

\section{Materials and Methods}

\section{Study Participants and Sample Collection}

A total of 426 pregnant women were prospectively admitted into Zhujiang Hospital (Guangzhou, China) for antenatal care during March 7-November 22, 2019. We excluded 14 from this cohort study because of insufficient samples for testing, incomplete clinical or experimental data, or invalid test results attributable to internal control failures. We included the remaining 412 samples in the prospective cohort study, in which direct culture, direct clinically validated PCR, and CRISPR-GBS tests were performed for each patient.

We conducted the validation cohort retrospectively, where we performed direct culture and CRISPR-GBS. For the purpose of validation, we included for enrichment culture 31 samples consisting of about one third each of dual-positive, dual-negative and discordant samples, according to the results of direct culture and CRISPR-GBS.

We collected vaginal-rectal swab specimens from the enrolled patients. Sample collection was reviewed and approved by the Zhujiang Hospital Ethics Committee Review Board. Informed consents were signed by patients or their surrogates.

\section{Cas13a Protein}

After codon optimization, we synthesized the open reading frame (ORF) of Cas13a and cloned it by using Gene Services (Genscript Biotech, https:/ / www.genscript.com). The Cas13a ORF expression vector was transfected into Escherichia coli BL21. We first grew transfected cells at $37^{\circ} \mathrm{C}$ and then incubated them with isopropyl $\beta$-d-1-thiogalactopyranoside at $16^{\circ} \mathrm{C}$. We purified proteins from lysed bacteria by using the Ni-NTA protocol (18) and stored aliquots of purified protein at $-80^{\circ} \mathrm{C}$.

\section{Strains and Human DNA}

We purchased the S. agalactiae (group B Streptococcus) strain from the American Type Culture Collection (ATCC13813). S. pneumoniae, S. pyogenes, S. mitis, Enterococcus faecalis, Acinetobacter baumannii, and Pseudomonas aeruginosa strains were donated by China's National Institutes for Food and Drug Control. We purchased another 2 species of bacteria, E. coli and Staphylococcus aureus, from China's General Microbiological Culture Collection Center. We purchased pure human DNA from Solarbio (http://www.solarbio. net), which we eluted in nuclease-free water.

\section{Oligos and gRNA}

Primer with an appended T7 promoter used in the recombinase polymerase amplification (RPA) for ato $B$ amplification were forward primer 5'-TAAT ACGA CTCA CTAT AGGG AATT GAAT GGAA TGAA CCAT TTGC AGCG AT-3' and reverse primer 5'AATA ATTC CTGA GCAG GCAT AAGG GTGT C-3'. We used sgRNA for Cas13 (5'-GGGG AUUU AGAC UACC CCAA AAAC GAAG GGGA CUAA AACU CUCU CUUC AGGA UAAU AAUG AUUA AAU-3') and ssRNA probe (5'-6-FAM-UUUUUC-BHQ1) for CRISPR detection after RPA amplification. Primer used in the nested PCR amplification for atoB amplification for round 1 were forward primer $5^{\prime}$-ACGG AAAA ACTA TTAA CAGA AACT CATA CT-3' and reverse primer 5'-AATA ATTC CTGA GCAG GCAT AAGG GTGT C-3' and for round 2 were forward primer 5'-CTCA TACT AAAA TATC GGAT TATG ATGC-3' and reverse primer 5'-AGGC ATAA GGGT GTCC GTAA GC-3'.

\section{DNA Rapid Extraction}

We eluted swabs with $1 \mathrm{~mL}$ of saline. We transferred $200 \mu \mathrm{L}$ of eluate to a new sterile, nucleasefree 1.5-mL tube. After a 5-minute centrifugation at $10,000 \times g$, we resuspended the pellet in lysis buffer consisting of $0.1 \%$ sodium dodecyl sulfate and $1 \%$ NP40. We added glass microbeads and used a Crystal Industries vortex mixer (https://crystalindustries.com) to disrupt the bacterial cell walls. We then heated samples at $99^{\circ} \mathrm{C}$ for $10 \mathrm{~min}$ and centrifuged them again at $14,000 \times g$. We used $2 \mu \mathrm{L}$ of supernatant as template for each subsequent assay for GBS detection.

\section{CRISPR-GBS}

The CRISPR-GBS test combines an RPA step and a subsequent $\mathrm{T} 7$ transcription and Cas13 detection step, as described previously (17). In brief, we incubated reactions containing $2 \mu \mathrm{L}$ of sample, $0.4 \mu \mathrm{M}$ of each primer, $1 \times$ reaction buffer, $14 \mathrm{mM}$ of magnesium acetate, and the RPA enzyme mix at $37^{\circ} \mathrm{C}$ for $30 \mathrm{~min}$. Then we added the amplification product to the CRISPR reaction mix, consisting of $33.3 \mathrm{nM}$ of gRNA, $66.7 \mathrm{nM}$ of Cas13, $5 \mathrm{mmol} / \mathrm{L}$ of each nucleotide triphosphate, $1 \mu \mathrm{L}$ of T7 RNA polymerase (New England Biolabs, https://www.neb. com) and $166 \mathrm{nM}$ of ssRNA reporter. We incubated the final reaction mix at $37^{\circ} \mathrm{C}$ and monitored it for fluorescence signal. We collected fluorescent signals by using an ABI7500 qPCR machine (ThermoFisher Scientific, https://www.thermofisher.com) for $20 \mathrm{~min}$. 


\section{Evaluation of Limit of Detection}

For the evaluation of limit of detection by the number of genomic copies, we purified DNA of the GBS strain (ATCC13813) and determined the concentration by using Qubit (ThermoFisher Scientific). We calculated the number of genomic copies by using the formula

$$
\text { copies }=\frac{\left(6.02 \times 10^{23}\right) \times\left(\text { concentration of DNA } \times 10^{-9}\right)}{(\text { length of DNA } \times 660)}
$$

We performed serial dilution with nuclease-free water to achieve desired concentrations. For the evaluation of limit of detection by CFU per $\mathrm{mL}$, we serially diluted a reference ATCC strain with known CFU with a negative sample to the desired titer before subjecting it to DNA extraction. Although accurate conversion is challenging, our and others' observations comparing DNA quantity and CFU counts showed that $1 \mathrm{CFU}$ equaled $\approx 3-5$ genome copies (data not shown) (19).

We used $2 \mu \mathrm{L}$ of extracted DNA at each titer as templates. We performed 10 replicates at each data point.

\section{Direct Culture and Enrichment Culture}

We eluted each swab with $1 \mathrm{~mL}$ of saline. For direct culture, we inoculated $200 \mu \mathrm{L}$ of eluate onto selective chromogenic GBS screening media (CHROMID Strepto B; bioMérieux, https://www.biomerieuxdiagnostics.com) and incubated it at $37^{\circ} \mathrm{C}$ for $24 \mathrm{~h}$ aerobically. We incubated negative plates for another $24 \mathrm{~h}$ before the final plate reading. For enrichment culture, we first inoculated $200 \mu \mathrm{L}$ of swab eluate into selective Todd Hewitt broth and incubated it at $37^{\circ} \mathrm{C}$ aerobically overnight. We then inoculated the enriched broth onto chromogenic Brilliance GBS agar (bioMérieux) by using the same experimental procedures as direct culture. We subjected all suspect colonies to Lancefield streptococcal grouping to confirm GBS.

\section{PCR and Nested PCR}

We performed the regular PCR testing by using a validated commercial GBS PCR kit (BEC, http:/ / www. biochainbj.com) according to the manufacturer's instructions. We performed the nested PCR assay in 2 successive rounds of amplification. The first round amplified a larger fragment of the ato $B$ gene for 35 cycles. We then subjected $2 \mu \mathrm{L}$ of the primary PCR product to the second amplification by using a nested set of primers targeting a shorter fragment as part of the first amplicon. We then purified the amplicons from the second round and subjected them to Sanger sequencing for validation. We considered positive only those samples that both yielded PCR products after the second round of amplification and had sequences validated by Sanger.

\section{Statistical Analysis}

We conducted comparative analysis by using Pearson $\chi^{2}$ test, Fisher exact test, or the Student $t$ test, where appropriate. We performed data analyses by using SPSS Statistics 22.0 (IBM, https:/ / www.ibm.com). We considered $p$ values $<0.05$ as statistically significant. All tests were 2-tailed unless indicated otherwise.

\section{Results}

\section{Development of CRISPR-GBS}

To address the challenges in clinical GBS screening, we aimed to develop a rapid, highly sensitive, and simple-to-use GBS assay by combining an RPA reaction with a CRISPR/Cas13 step for target detection (17). We established a rapid extraction method for high efficiency GBS DNA extraction by combining chemical, heat, and bead beating-based cell wall disruption, which eliminated the need for any column and organic solvents (Figure 1; Appendix Figure 1, https://wwwnc.cdc.gov/EID/article/27/9/200091-App1.pdf). This strategy takes advantage of both the polymerase-mediated DNA amplification and the CRISPR/Cas-mediated enzymatic signal amplification for greater sensitivity. Moreover, the rapid extraction and isothermal nature of such an assay eliminated the demand for sophisticated instruments such as thermal cyclers.

We chose the thiolase (atoB) gene as the target region in this assay because it is highly conserved and specific for the GBS genome (20). We screened multiple sets of RPA primers and CRISPR gRNAs targeting different regions within atoB (Appendix Table 2, Figure 2). The set that showed the best overall performance of sensitivity and specificity was then used in this study for assay optimization and clinical diagnostic evaluation.

We then sought to determine the analytical sensitivity by serial dilutions of GBS with negative swabs at various counts of CFU per mL. CRISPR-GBS managed to detect samples at $30 \mathrm{CFU} / \mathrm{mL}$ in 6 of 10 runs and at $60 \mathrm{CFU} / \mathrm{mL}$ in all 10 replicates (Figure 2, panel A). We further assessed the limit of detection of CRISPRGBS by titrations of copies per reaction. The CRISPR assay consistently detected 5 copies of GBS in 10 of 10 runs and 2 copies in 4 of 10 replicates (Figure 2, panel B). These data indicate that CRISPR-GBS could detect a low number of genome copies or $\approx 50 \mathrm{CFU} / \mathrm{mL}$ and 


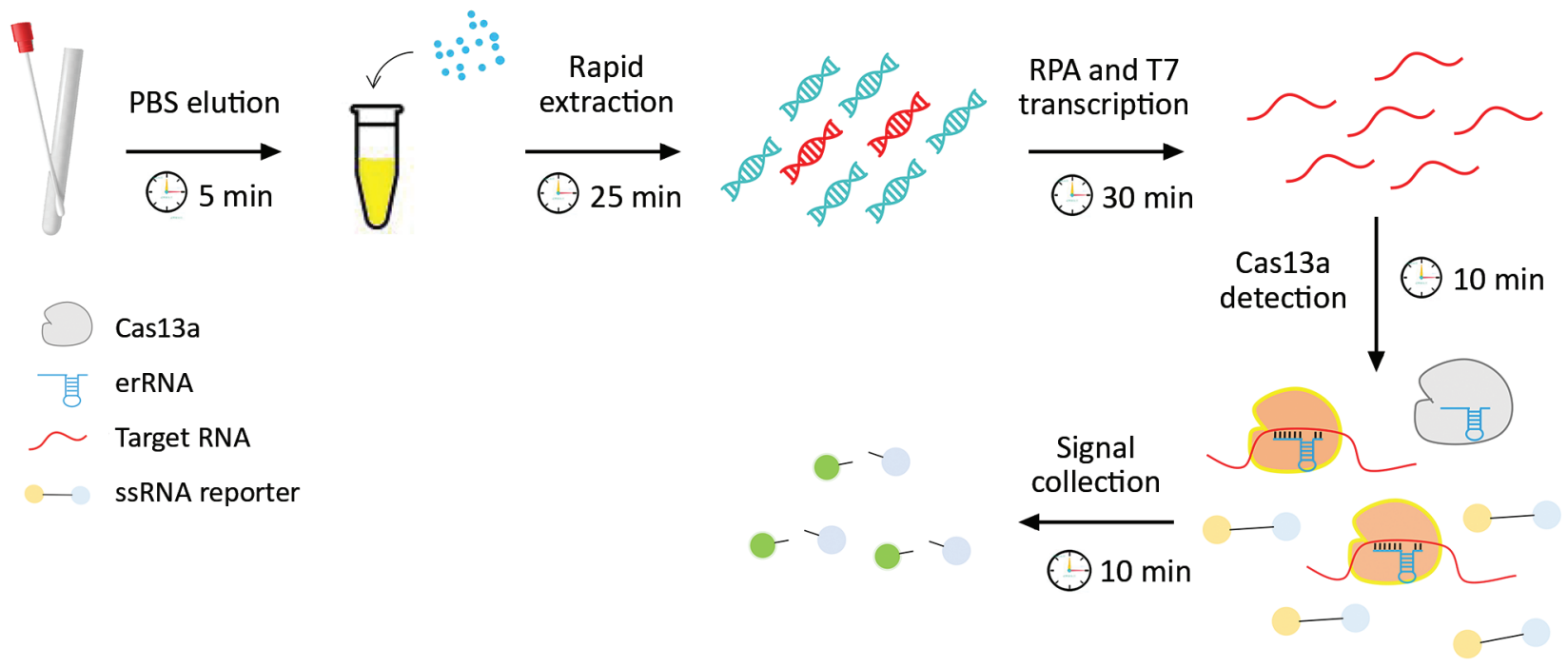

Figure 1. Schematic diagram of CRISPR-based diagnostic for rapid GBS screening. Swab samples are first eluted and followed by a rapid DNA extraction step where the bacterial cell walls are disrupted by a combination of chemical, physical, and heating effects. The extracted DNA is then subjected to the CRISPR/Cas reaction. The collateral nuclease activity of Cas proteins are activated upon specific binding of gRNA to the ato $B$ gene. Fluorescent signal produced from cleaved probes is captured and indicates the presence of GBS. GBS, group B Streptococcus. gRNA, guide RNA; ssRNA, single-stranded RNA.

is more sensitive than most of the commercially available US Food and Drug Administration-approved GBS assays, such as GeneXpert GBS (300 CFU/mL) (Cepheid, https://www.cepheid.com), BD Max GBS (1,000 CFU/mL) (BD, https://www.bd.com), Quidel Solana GBS $\left(2.6 \times 10^{5} \mathrm{CFU} / \mathrm{mL}\right)$ (Quidel, https:// www.quidel.com), and AmpliVue GBS $\left(1.4 \times 10^{6}\right.$ CFU/mL) (Quidel) (20-22).

With such a high sensitivity of CRISPR-GBS, we set out to confirm its specificity. For this purpose, we assayed DNA from humans and a panel of bacteria, including bacteria in the same genus (e.g., S. pneumonia, S. pyogenes, and S. mitis), microbes commonly found in vaginal swabs (e.g., E. coli, Staphylococcus aureus, and Enterococcus faecalis), and bacteria commonly found in nosocomial infections (e.g., Acinetobacter baumannii and Pseudomonas aeruginosa) (23). Of note, none of these interference samples triggered a falsepositive reaction (Figure 2, panel C). Altogether, these analytical evaluations suggest that CRISPR-GBS, with its great sensitivity and specificity, is a promising molecular assay for GBS detection.

\section{Clinical Diagnostic Evaluation of CRISPR-GBS}

After the analytical study, we further assessed the diagnostic potential of CRISPR-GBS in settings of clinical screening. A total of 426 pregnant women with a median age of 29 years (20-47 years) were enrolled in this cohort study. Sample collection was performed at 34-38 weeks of gestation. Among these patients,
14 were excluded because of invalid test results, an insufficient specimen, or both. The remaining 412 patients were tested for GBS by culture, PCR, and CRISPR-GBS on their direct swab samples. We found no significant differences between patients who were negative or positive for GBS on the basis of patient age or weeks of gestation (Appendix Table 1).

When we conducted the CRISPR-GBS assay, we included a positive control of GBS DNA and a no-template control in parallel for each run. We used a fluorescent signal from no-template control normalize the signal of other samples in the same run to calculate the corresponding fold changes. We noticed clear distinctions in signal patterns of the reactions. Specifically, the fluorescent signal curve either remained flat (e.g., the no-template control runs) or had a distinguishable takeoff from the baseline (e.g., the positive control runs) (Figure 3, panel A). To determine the cutoff value as fold-changes for the CRISPR-GBS results, we first separated all the runs into a tentatively positive group and a tentatively negative group according to these distinct patterns. We then analyzed the cutoff values. The tentatively positives had fold changes ranging from 3.9 to 90.3 (median 26.3), whereas the tentatively negatives ranged from 0.5 to 2.9 (median 1.5) (Figure 3, panel B; Appendix Figure 3). Therefore, we were able to set the cutoff value at 3.5 for complete separation of the 2 groups. Consistently, this cutoff was further confirmed by the receiver operating characteristic analysis for optimal sensitivity and specificity (data not shown). 
To evaluate the diagnostic performances of different methodologies for GBS detection, we began by comparing direct culture and PCR. We found a concordance of $97.1 \%$ between these 2 traditional methods. Specifically, only $5(1.2 \%)$ of 412 culturepositive and $7(1.7 \%)$ of 412 PCR-positive cases were missed by the other test. When culture was used as the reference standard, PCR demonstrated a sensitivity of $90.9 \%$ (50/55 results) and specificity of $98.0 \%$ (350/357 cases).

We further assessed the CRISPR-GBS test in comparison with direct culture and the PCR-based assay (Table; Figure 4). When the comparison was made separately, CRISPR-GBS was able to detect most of the positive samples by either reference method, with a sensitivity of $94.5 \%$ (52/55 cases) compared with culture and $94.7 \%$ (54/57 cases) compared with PCR. When we included only the 400 cases with concordant culture and PCR results in the analysis, CRISPR identified $94.0 \%(47 / 50)$ of the positive results and offered a negative predictive value of $99.1 \%$ (320/323 cases).

Among the cases reported negative by culture, PCR, or both, we also found $\approx 10 \%$ of them to be positive by CRISPR, which included 37 of 357 culturenegative cases, 35 of 355 PCR-negative cases, and 30 of 350 dual-negative cases (i.e., by culture and PCR). These data indicate a greater sensitivity or a lower specificity of CRISPR-GBS.


Figure 2. Analytical assessment of the sensitivity and specificity of CRISPR-based diagnostic for rapid GBS screening. Evaluation was performed by testing contrived negative swab samples with indicated CFUs of GBS (A), different copy numbers of GBS genomic DNA (B), and various microbes as interfering materials (C). GBS, group B Streptococcus. A. baumannii, Acinetobacter baumannii; E. coli, Escherichia coli; E. faecalis, Enterococcus faecalis; hDNA, human DNA; P.aeruginosa, Pseudomonas aeruginosa; S. aureus, Staphylococcus aureus; S. mitis, Streptococcus mitis; S. pneumoniae, Streptococcus pneumoniae; S. pyogenes, Streptococcus pyogenes. 


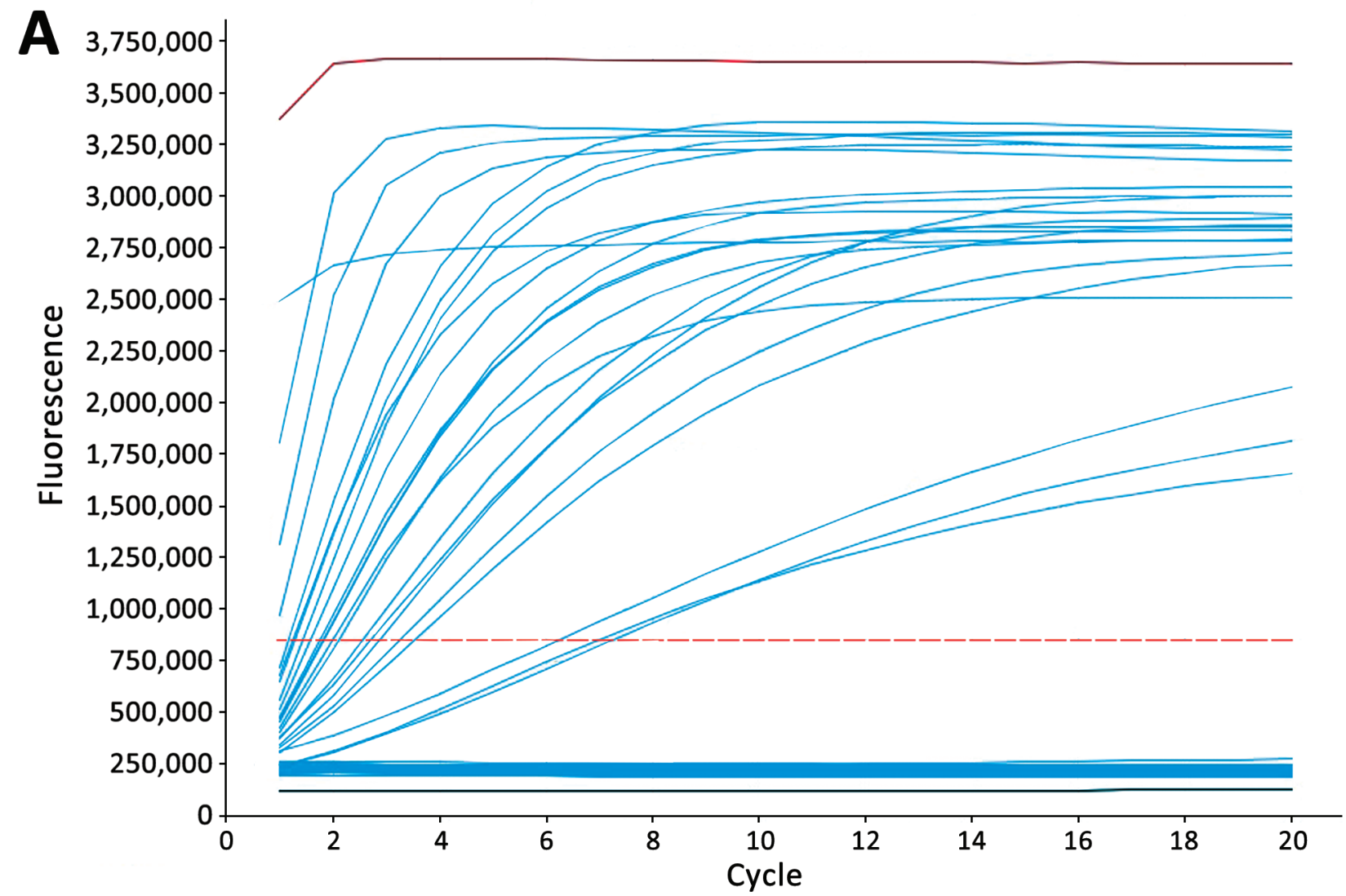

B

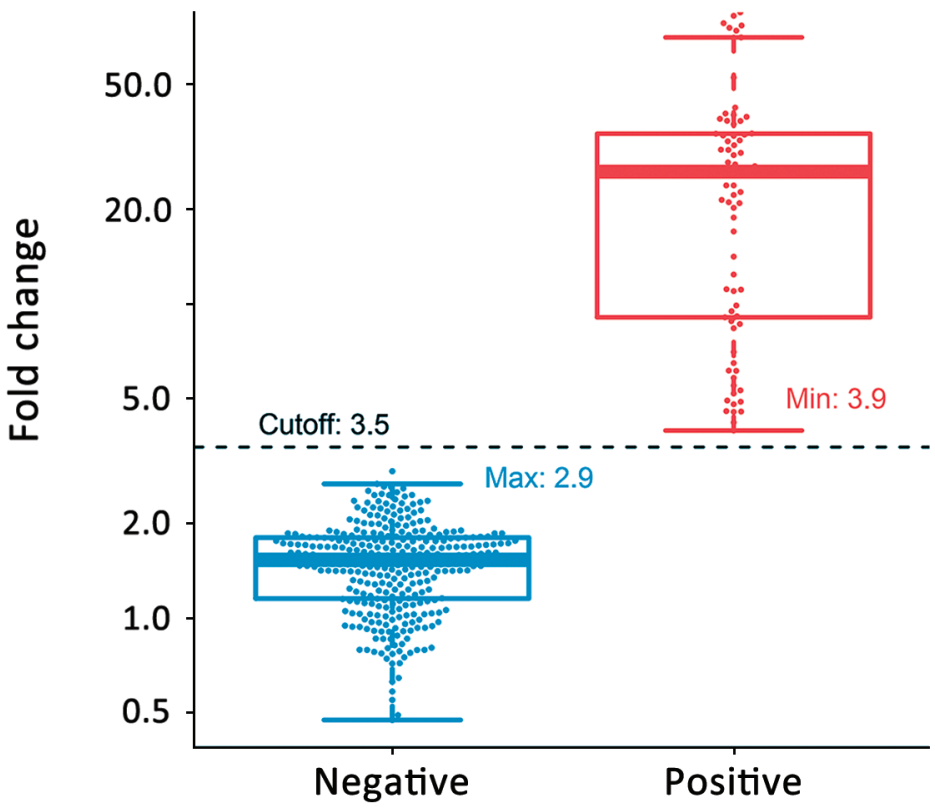

Figure 3. Determination of assay cutoff for CRISPR-based diagnostic for rapid GBS screening. A) Representative signal curves produced by CRISPR-GBS. A positive control (red), a negative control (black), and 85 clinical samples (blue) are shown with distinct curve patterns (take-off vs. flat). B) Fold-change values by CRISPR-GBS obtained from our prospective cohort: positive (with take-off signal curves in red) and negative (flat curves in blue). A cutoff of 3.5 was set and is indicated in black dashed line. GBS, group B Streptococcus. Lines from the bottom to the top of box-and-whisker plots refer to minimum, first quartile (Q1), median, third quartile (Q3), and maximum number of the dataset.

We designed and conducted additional validation studies in an attempt to validate the improved sensitivity of CRISPR-GBS. We developed a nested PCR-Sanger assay targeting the atoB gene, in which we performed 2 successive rounds of PCR in a nested manner to achieve greater amplification sensitivity compared with regular single-round PCR reactions. We then subjected the amplicons to Sanger sequencing for further validation. With this nested PCR assay, we tested the 30 specimens that were only positive by 
Table. Positive and negative agreement of CRISPR-based diagnostic for rapid group B Streptococcus screening versus different reference standards*

\begin{tabular}{|c|c|c|c|c|c|c|c|}
\hline \multirow[b]{3}{*}{ Assay and result } & \multirow{2}{*}{\multicolumn{3}{|c|}{ CRISPR-GBS }} & \multicolumn{4}{|c|}{$\%(95 \% \mathrm{Cl})$} \\
\hline & & & & \multirow[b]{2}{*}{ Sensitivity } & \multirow[b]{2}{*}{ Specificity } & \multirow{2}{*}{$\begin{array}{c}\text { Positive predictive } \\
\text { value }\end{array}$} & \multirow{2}{*}{$\begin{array}{c}\text { Negative } \\
\text { predictive value }\end{array}$} \\
\hline & Positive & Negative & Total & & & & \\
\hline \multicolumn{8}{|l|}{ Direct culture } \\
\hline Positive & 52 & 3 & 55 & $94.5(83.9-98.6)$ & 89.6 (85.9-92.5) & $58.4(47.5-68.6)$ & $99.1(97.1-99.8)$ \\
\hline Negative & 37 & 320 & 357 & & & & \\
\hline Total & 89 & 323 & 412 & & & & \\
\hline \multicolumn{8}{|l|}{ PCR } \\
\hline Positive & 54 & 3 & 57 & 94.7 (84.5-98.6) & $90.1(86.4-92.9)$ & 60.7 (49.7-70.7) & $99.1(97.1-99.8)$ \\
\hline Negative & 35 & 320 & 355 & & & & \\
\hline Total & 89 & 323 & 412 & & & & \\
\hline \multicolumn{8}{|c|}{ Direct culture and PCR } \\
\hline Positive & 47 & 3 & 50 & $94.0(82.5-98.4)$ & $91.4(87.9-94.0)$ & $61.0(49.2-71.7)$ & $99.0(97.1-99.8)$ \\
\hline Negative & 30 & 320 & 350 & & & & \\
\hline Total & 77 & 323 & 400 & & & & \\
\hline \multicolumn{8}{|l|}{ Enriched culture } \\
\hline Positive & 22 & 0 & 22 & $100(81.5-100.0)$ & $100(62.9-100.0)$ & $100(81.5-100.0)$ & $100(62.9-100.0)$ \\
\hline Negative & 0 & 9 & 9 & & & & \\
\hline Total & 22 & 9 & 31 & & & & \\
\hline
\end{tabular}

CRISPR-GBS but negative by both direct culture and regular PCR in our cohort. We were able to confirm 15 of 30 discordant cases (Figure 4, panel A). These data supported the previous findings and again indicate higher sensitivity of CRISPR-GBS compared with direct culture or PCR.

To further rule out the possibility of false-positive results, we set up a retrospective validation study and compared the sensitivity of CRISPR-GBS with enrichment culture, which had been shown to be more sensitive than direct culture $(5,24)$. The validation cohort of 31 patients consisted of 13 CRISPR-positive and direct culture-positive, 10 CRISPR-positive and direct culture-negative, and 8 CRISPR-negative and direct culture-negative samples. We tested each sample by direct culture, enriched culture, and CRISPR-GBS both before and after broth enrichment. We performed enriched culture by overnight culture in selective broth, followed by inoculation onto blood agar. We found that the samples that were negative by both direct culture and CRISPR originally would remain negative even after broth enrichment. However, of the 10

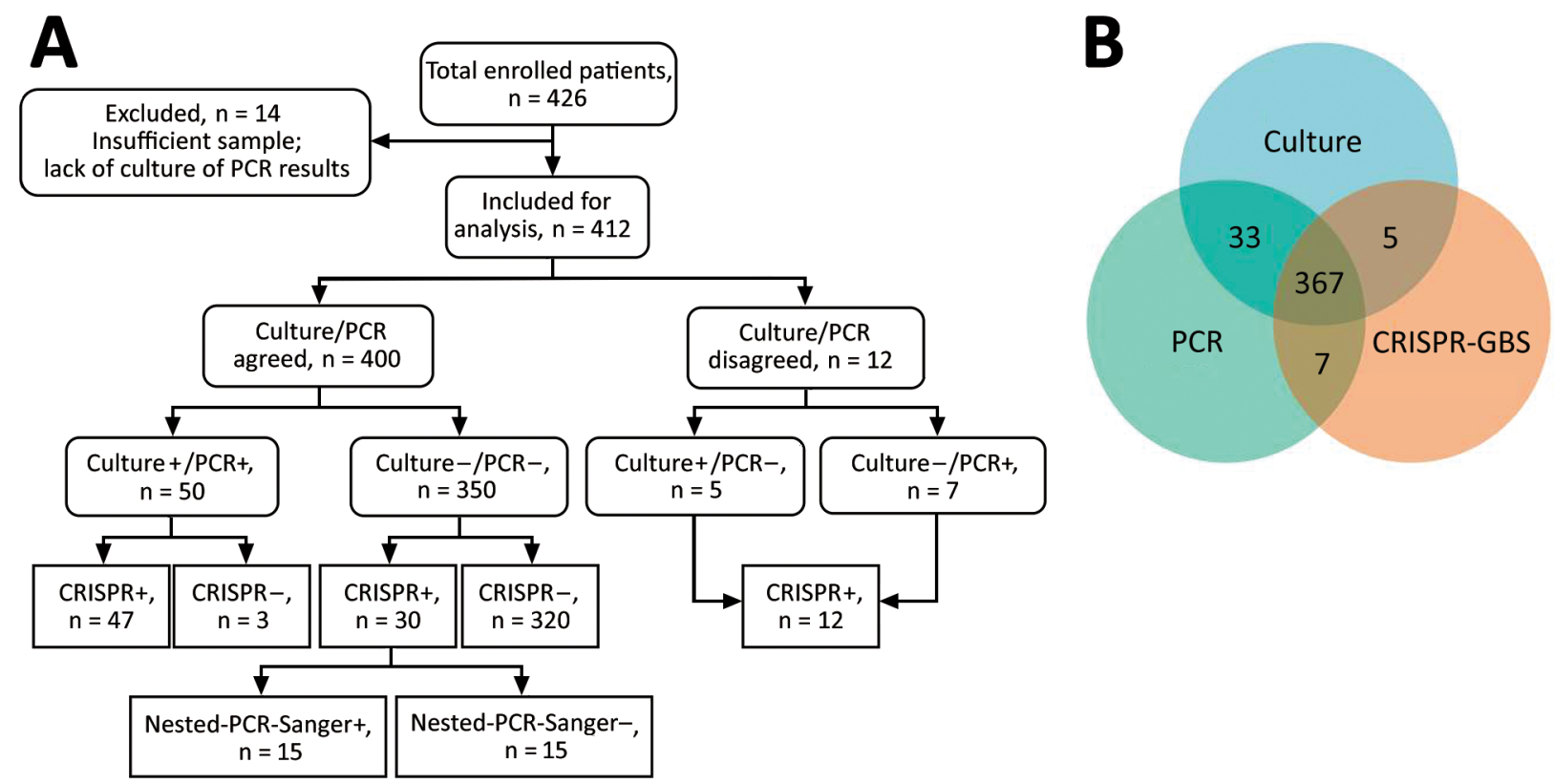

Figure 4. Overview and summary of the prospective cohort study assessing CRISPR-GBS. A) Study enrollment and result summary as categorized by agreements between different tests. B) Venn diagram demonstrating the overall concordance and discordance among direct culture, regular PCR, and CRISPR-GBS in the cohort. CRISPR-GBS, CRISPR-based diagnostic for rapid group B Streptococcus screening. 
cases that were positive by CRISPR but negative by direct culture, adding the broth enrichment step yielded positive results in $90 \%$ of those cases (Figure 5). These results validated the greater sensitivity of CRISPR and suggested that the testing direct swabs by CRISPRGBS conferred comparable sensitivity as enrichment culture. In our antepartum cohort of 412 pregnant women, the prevalence of GBS carriage was the highest by CRISPR at $21.6 \%(89 / 412)$ and was similar by culture (13.3\% [55/412]), and PCR (13.8\% [57/412]).

When we compared turnaround time, we found that the CRISPR-GBS test required an average of $<1.5$ hours, which includes 30 minutes of rapid DNA extraction, 30 minutes for DNA amplification by RPA, and 20 minutes for Cas13 detection. This turnaround time is a considerable advantage over those for conventional culture-based (24-60 hours) and PCR-based $(\approx 2.5$ hours for a regular PCR assay and much longer for nested PCR-Sanger) methods.

\section{Discussion}

We developed and demonstrated a CRISPR-based assay that offered short turn-around time and great sensitivity, which makes it a potential rapid, pointof-care assay for intrapartum GBS diagnosis, even in low-resource settings. Debates have occurred over approaches of preventing neonatal diseases caused by GBS infection (25). However, both of the 2 commonly used conventional strategies (i.e., risk-based screening or late antenatal microbiologic testing) have their own limitations $(3,26)$. A point-of-care, rapid intrapartum GBS diagnosis at the onset of labor or membrane rupture is highly desired clinically because it would enable more accurate antibiotic prophylaxis and better antimicrobial stewardship (5). Successful development of such a diagnostic has been hindered by its requirement for a combination of short turnaround time, high diagnostic performance, low technical complexity, and low instrument requirement. In our study, we took advantage of the programmable
CRISPR/Cas system for GBS detection. The CRISPRGBS assay as established and demonstrated in our study takes $<1.5$ hours to complete, has a sensitivity comparable to enriched culture, and does not require any sophisticated instruments. These features illustrate its great potential to be an onsite, rapid diagnostic for intrapartum GBS screening. Given the low complexity of the CRISPR-GBS assay established in our study, integration of the entire testing into a compact desktop instrument for an automated sample-inreport-out assay is highly feasible.

In our prospective study, we found the prevalence of GBS in our cohort to be slightly higher than $20 \%$ by CRIPSR. Although studies have shown differential prevalence between rectal and vaginal screening, the question of whether this could be caused by a lack of assay sensitivity for detecting borderline bacterial level remains controversial $(1,24,27)$. In current clinical practice, vaginal-rectal swab specimens are commonly collected for optimized GBS detection, despite reported discomfort or even pain associated with rectal swabs $(28,29)$. Determining whether patients could be spared the discomfort of rectal specimens without compromising the results with a more sensitive assay would be worthwhile. With this sensitive and rapid CRISPR assay, further studies are also warranted to evaluate its diagnostic and clinical value as an intrapartum assay by comparing it to antepartum culture (30).

Apart from GBS diagnosis, obtaining the information on drug susceptibility is also of great clinical value. For instance, recent reports have showed a trend of increased erythromycin and clindamycin resistance internationally (31-33). Genotypic analysis has been proven to have great predictive value for drug resistance. Given the highly sensitive nature of this CRISPR diagnostic technology, it holds the potential to simultaneously detect genes related to drug susceptibility (34). An expanded CRISPR-GBS assay would be able to not only diagnose GBS colonization

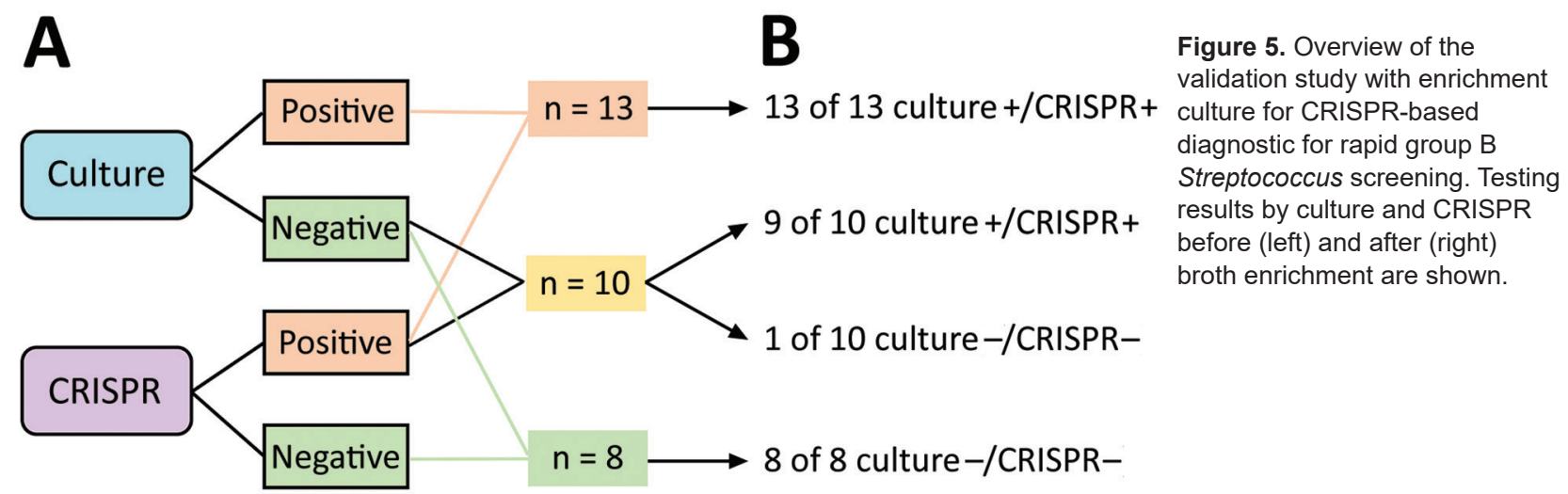


but also provide genetic insight into drug susceptibility for first-line antibiotics. On the basis of the proofof-principle demonstrated in our study for directfrom-swab testing, rapid CRISPR detection of both pathogen and drug sensitivities would permit the precise approach to identification of GBS colonization and prevention of related neonatal diseases.

Because GBS is an important infection agent for multiple invasive infectious diseases such as meningitis, CRISPR-GBS could also be a promising tool for potentially much wider applications. A future multicenter study with a larger cohort would provide a more thorough evaluation of its diagnostic value, including its performance under different clinical settings.

In summary, the CRISPR-based rapid GBS assay we established in this study exhibits great diagnostic performance for GBS colonization under analytical and clinical settings. This novel test offers improved diagnostic performance over culture- and PCR-based assays and represents a novel option for potential rapid, point-of-care GBS screening.

This study was supported by the National Key Research and Development Project (grant no. 2017YFC1200800) and the Research and Development Projects in Key Areas of Guangdong Province (grant no. 2018B020241002).

A patent application covering the specific primers and crRNA described in the CRISPR-GBS assay has been filed (W.Z. and T.X). All other findings and reagents are in the public domain. No other authors have ownership, patent, royalty, or other financial interest in the technique or reagents to declare.

\section{About the Author}

Dr. Jiang is Senior Technologist at Zhujiang Hospital, Southern Medical University, Guangzhou, China. His primary research interests are pathogenic mechanisms of clinical pathogens and the development of novel rapid diagnostic assays.

\section{Reference}

1. Dillon HC Jr, Gray E, Pass MA, Gray BM. Anorectal and vaginal carriage of group B streptococci during pregnancy. J Infect Dis. 1982;145:794-9. https:/ / doi.org/10.1093/ infdis/145.6.794

2. American Academy of Pediatrics Committee on Infectious Diseases and Committee on Fetus and Newborn. Guidelines for prevention of group B streptococcal (GBS) infection by chemoprophylaxis. Pediatrics. 1992;90:775-8.

3. Melin P. Neonatal group B streptococcal disease: from pathogenesis to preventive strategies. Clin Microbiol Infect. 2011;17:1294-303. https://doi.org/10.1111/ j.1469-0691.2011.03576.x
4. Phares CR, Lynfield R, Farley MM, Mohle-Boetani J, Harrison LH, Petit S, et al.; Active Bacterial Core surveillance/Emerging Infections Program Network. Epidemiology of invasive group B streptococcal disease in the United States, 1999-2005. JAMA. 2008;299:2056-65. https://doi.org/10.1001/jama.299.17.2056

5. Verani JR, McGee L, Schrag SJ; Division of Bacterial Diseases, National Center for Immunization and Respiratory Diseases, Centers for Disease Control and Prevention. Prevention of perinatal group B streptococcal disease - revised guidelines from CDC, 2010. MMWR Recomm Rep. 2010;59(RR-10):1-36.

6. The Royal Australian and New Zealand College of Obstetricians and Gynaecologists. Maternal group B Streptococcus in pregnancy: screening and management (C-Obs 19). 2019 [cited 2021 Jul 19]. https:/ / ranzcog. edu.au/RANZCOG_SITE/media/RANZCOG-MEDIA/ Women $\% 27 \mathrm{~s} \% 20 \mathrm{Health} /$ Statement $\% 20$ and $\%$ 20guidelines/ Clinical-Obstetrics/Maternal-Group-B-Streptococcus-inpregnancy-screening-and-management-(C-Obs-19).pdf

7. Schrag SJ, Zywicki S, Farley MM, Reingold AL, Harrison LH, Lefkowitz LB, et al. Group B streptococcal disease in the era of intrapartum antibiotic prophylaxis. N Engl J Med. 2000;342:15-20. https:/ / doi.org/10.1056/ NEJM200001063420103

8. Hansen SM, Uldbjerg N, Kilian M, Sørensen UB. Dynamics of Streptococcus agalactiae colonization in women during and after pregnancy and in their infants. J Clin Microbiol. 2004;42:83-9. https:// doi.org/10.1128/ JCM.42.1.83-89.2004

9. Valkenburg-van den Berg AW, Houtman-Roelofsen RL, Oostvogel PM, Dekker FW, Dörr PJ, Sprij AJ. Timing of group B streptococcus screening in pregnancy: a systematic review. Gynecol Obstet Invest. 2010;69:174-83. https:// doi.org/10.1159/000265942

10. Boyer KM, Gadzala CA, Kelly PD, Burd LI, Gotoff SP. Selective intrapartum chemoprophylaxis of neonatal group B streptococcal early-onset disease. II. Predictive value of prenatal cultures. J Infect Dis. 1983;148:802-9. https://doi.org/10.1093/infdis/148.5.802

11. Yancey MK, Schuchat A, Brown LK, Ventura VL, Markenson GR. The accuracy of late antenatal screening cultures in predicting genital group B streptococcal colonization at delivery. Obstet Gynecol. 1996;88:811-5. https://doi.org/10.1016/0029-7844(96)00320-1

12. Hwang WY, Fu Y, Reyon D, Maeder ML, Tsai SQ, Sander JD, et al. Efficient genome editing in zebrafish using a CRISPR-Cas system. Nat Biotechnol. 2013;31:227-9. https:// doi.org/10.1038/nbt.2501

13. Wu Y, Liang D, Wang Y, Bai M, Tang W, Bao S, et al. Correction of a genetic disease in mouse via use of CRISPR-Cas9. Cell Stem Cell. 2013;13:659-62. https:/ / doi.org/ 10.1016/j.stem.2013.10.016

14. Knott GJ, Doudna JA. CRISPR-Cas guides the future of genetic engineering. Science. 2018;361:866-9. https://doi.org/10.1126/science.aat5011

15. Chen JS, Ma E, Harrington LB, Da Costa M, Tian X, Palefsky JM, et al. CRISPR-Cas12a target binding unleashes indiscriminate single-stranded DNase activity. Science. 2018;360:436-9. https://doi.org/10.1126/science.aar6245

16. Harrington LB, Burstein D, Chen JS, Paez-Espino D, Ma E, Witte IP, et al. Programmed DNA destruction by miniature CRISPR-Cas14 enzymes. Science. 2018;362:839-42. https://doi.org/10.1126/science.aav4294

17. Myhrvold C, Freije CA, Gootenberg JS, Abudayyeh OO, Metsky HC, Durbin AF, et al. Field-deployable viral 
diagnostics using CRISPR-Cas13. Science. 2018;360:444-8. https://doi.org/10.1126/science.aas8836

18. Crowe J, Döbeli H, Gentz R, Hochuli E, Stüber D, Henco K. 6xHis-Ni-NTA chromatography as a superior technique in recombinant protein expression/purification. Methods Mol Biol. 1994;31:371-87.

19. Parham NJ, Picard FJ, Peytavi R, Gagnon M, Seyrig G, Gagné PA, et al. Specific magnetic bead based capture of genomic DNA from clinical samples: application to the detection of group B streptococci in vaginal/anal swabs. Clin Chem. 2007;53:1570-6. https://doi.org/10.1373/ clinchem.2007.091389

20. Miller SA, Deak E, Humphries R. Comparison of the AmpliVue, BD Max System, and Illumigene molecular assays for detection of group B Streptococcus in antenatal screening specimens. J Clin Microbiol. 2015;53:1938-41. https://doi.org/10.1128/JCM.00261-15

21. Park JS, Cho DH, Yang JH, Kim MY, Shin SM, Kim EC, et al. Usefulness of a rapid real-time PCR assay in prenatal screening for group B streptococcus colonization. Ann Lab Med. 2013;33:39-44. https:/ / doi.org/10.3343/alm.2013.33.1.39

22. Berry GJ, Zhang F, Manji R, Juretschko S. Comparison of the Panther Fusion and BD MAX Group B Streptococcus (GBS) assays for detection of GBS in prenatal screening specimens. J Clin Microbiol. 2019;57:e1034-19. https:/ / doi.org/ 10.1128/JCM.01034-19

23. Ke D, Ménard C, Picard FJ, Boissinot M, Ouellette M, Roy $\mathrm{PH}$, et al. Development of conventional and real-time PCR assays for the rapid detection of group B streptococci. Clin Chem. 2000;46:324-31. https:/ /doi.org/10.1093/ clinchem/46.3.324

24. Platt MW, McLaughlin JC, Gilson GJ, Wellhoner MF, Nims LJ. Increased recovery of group B streptococcus by the inclusion of rectal culturing and enrichment. Diagn Microbiol Infect Dis. 1995;21:65-8. https:/ / doi.org/ 10.1016/0732-8893(95)00022-3

25. Davies HD. Preventing group B streptococcal infections: new recommendations. Can J Infect Dis. 2002;13:232-5. https:/ / doi.org/10.1155/2002/352613

26. Puopolo KM, Lynfield R, Cummings JJ; Committee on Fetus and Newborn; Committee on Infectious Diseases. Management of infants at risk for group B streptococcal disease. Pediatrics. 2019;144:e20191881. https://doi.org/10.1542/ peds.2019-1881

27. Philipson EH, Palermino DA, Robinson A. Enhanced antenatal detection of group B streptococcus colonization.
Obstet Gynecol. 1995;85:437-9. https://doi.org/10.1016/ 0029-7844(94)00412-7

28. Jamie WE, Edwards RK, Duff P. Vaginal-perianal compared with vaginal-rectal cultures for identification of group B streptococci. Obstet Gynecol. 2004;104:1058-61. https:/ / doi.org/10.1097/01.AOG.0000144120.20312.ed

29. Orafu C, Gill P, Nelson K, Hecht B, Hopkins M. Perianal versus anorectal specimens: is there a difference in group B streptococcal detection? Obstet Gynecol. 2002;99:1036-9. https://doi.org/10.1097/ 00006250-200206000-00015

30. Iams JD, O'Shaughnessy R. Antepartum versus intrapartum selective screening for maternal group B streptococcal colonization. Am J Obstet Gynecol. 1982;143:153-6. https:/ / doi.org/10.1016/0002-9378(82)90645-7

31. Guo Y, Deng X, Liang Y, Zhang L, Zhao GP, Zhou Y. The draft genomes and investigation of serotype distribution, antimicrobial resistance of group B Streptococcus strains isolated from urine in Suzhou, China. Ann Clin Microbiol Antimicrob. 2018;17:28. https:/ / doi.org/10.1186/ s12941-018-0280-y

32. Gao K, Guan X, Zeng L, Qian J, Zhu S, Deng Q, et al. An increasing trend of neonatal invasive multidrugresistant group B streptococcus infections in southern China, 2011-2017. Infect Drug Resist. 2018;11:2561-9. https://doi.org/10.2147/IDR.S178717

33. Tsai MH, Hsu JF, Lai MY, Lin LC, Chu SM, Huang HR, et al. Molecular characteristics and antimicrobial resistance of group b Streptococcus strains causing invasive disease in neonates and adults. Front Microbiol. 2019;10:264. https:/ / doi.org/10.3389/fmicb.2019.00264

34. Campisi E, Rosini R, Ji W, Guidotti S, Rojas-López M, Geng $\mathrm{G}$, et al. Genomic analysis reveals multi-drug resistance clusters in group B Streptococcus CC17 hypervirulent isolates causing neonatal invasive disease in southern mainland China. Front Microbiol. 2016;7:1265. https:/ / doi.org/ 10.3389/fmicb.2016.01265

Address for correspondence: Teng Xu, Vision Medicals Center for Medical Research, 31 Kefeng Ave, Bldg G10, Unit 301, Guangzhou 510000, China; email: txu@visionmedicals.com; Shilei Pan, Department of Prenatal Diagnosis, Zhujiang Hospital, Southern Medical University, 253 Middle Gongye Ave, Guangzhou 510282, China; email: 13602882918@163.com 